\title{
Editorial: Advances in Mobile, Edge and Cloud Computing
}

\author{
Xiaowen $\mathrm{Chu}^{1} \cdot$ Hongbo Jiang ${ }^{2} \cdot \mathrm{Bo} \mathrm{Li}^{3} \cdot$ Dan Wang ${ }^{4} \cdot$ Wei Wang $^{3}$ \\ Published online: 26 September 2020 \\ (C) Springer Science+Business Media, LLC, part of Springer Nature 2020
}

\section{Editorial:}

Emerging mobile applications exhibit heterogeneous requirements on the computing power, communication bandwidth, security and privacy. Given the restriction on the computing capability on battery-operated mobile devices, a variety of offloading techniques have been designed to leverage the abundant computing resources available on cloud servers. Mobile Cloud Computing provides enormous computing and storage resources for mobile applications that can tolerate a certain level of network delay, while Mobile Edge Computing offers an intelligent platform to enhance mobile devices' capabilities and improve the Quality of Service of mobile applications. Both Mobile Cloud Computing and Mobile Edge Computing are key enabling paradigms for emerging mobile applications in Internet of Things (IoT), smart grids, robotics, crowd sensing, etc. New research challenges arise due to the heterogeneity of computing and network resources across the mobile devices, edge servers, and remote data centers.

Aiming to address the challenges in resource planning and management, scheduling, networking, security and privacy in mobile edge and cloud computing, this special issue features nine selected articles with high quality. The first article, "Batch Auction Design for Cloud Container Services", proposes efficient market mechanisms for container based cloud service. To simultaneously achieve incentive compatibility, computational efficiency, and economic efficiency, the

Xiaowen Chu

chxw@Comp.HKBU.Edu.HK

1 Hong Kong Baptist University, 224 Waterloo Rd, Kowloon Tong, Hong Kong

2 Hunan University, 2 Lushan S Rd, Yuelu District, Changsha, Hunan, China

3 The Hong Kong University of Science and Technology, Clear Water Bay, Hong Kong

4 The Hong Kong Polytechnic University, 11 Yuk Choi Rd, Hung Hom, Hong Kong authors leveraged compact exponential integer linear program, posted price auction, and primal-dual algorithms. Theoretical analysis and trace-driven empirical evaluation verify the efficacy of the proposed container auction algorithms.

The second article entitled "Computing Cost Optimization for Multi-BS in MEC by Offloading" presents two novel task offloading strategies to optimally assign IoT tasks to multiple edge servers deployed at base stations. The offloading strategies, which are based on Genetic Algorithm, offload as many tasks as possible while taking into account both the computation overhead and energy consumption. Extensive numerical experiments are presented to demonstrate the effectiveness of the proposed offloading strategies.

In the next article with the title "Effective Mobile Target Searching Using Robots", the authors studied a robotics application in which a swarm of Unmanned Aerial Vehicles (UAVs) collaboratively search for a set of targets. They proposed a divide-and-conquer approach with two searching strategies, namely the circuit strategy and the rebound strategy, and further proved that the expected searching latency of the circuit strategy is upper bounded by a factor that is linear to the number of grid cells of the search region. The authors conducted simulations to verify that the circuit strategy with the failure handling mechanism can achieve the best search effectiveness.

The fourth article entitled "A Reinforcement Learning based Data Storage and Traffic Management in InformationCentric Data Center Networks" studies the problem of data storage and traffic management in the context of InformationCentric Data Center Networks (ICDCN) and proposes a distributed multi-agent Q-learning-based scheme to support efficient data storage and traffic management in ICDCN. The authors carried out simulations to show that the proposed algorithm can effectively balance the load of nodes and improve the system throughput while reducing the transmission completion time.

The fifth article, "Neighborhood-aware Mobile Hub: An Edge Gateway with Leader Election Mechanism for Internet of Mobile Things," proposes a neighborhood-aware leader- 
election mechanism to judiciously determine which Mobile Hub should be selected to monitor a smart mobile object. The proposed mechanism addresses a number of challenges posed by the mobility of both the hub nodes and the object nodes, by jointly considering their battery level and network status. The authors conducted extensive testbed experiments to demonstrate the effectiveness of the proposed mechanism.

The sixth article entitled "Privacy-preserving and Utilityaware Participant Selection for Mobile Crowd Sensing" proposes a novel privacy-preserving mobile crowd sensing scheme for participant selection that has been presented to collect the well-measured sensing data from the set of participants to achieve securely optimal data utility within the total budget and without disclosing any information. The scheme significantly improves the privacy of the participant in the selection process. It enables SP to build a secure and efficient MCS system that supports the privacy of users and obtains optimized data utility from participants.

The seventh article, "Raven: Scheduling Virtual Machine Migration during Datacenter Upgrades with Reinforcement Learning," studies migrating virtual machines (VMs) to alternative physical machines in data centers during the upgrade of all the physical machines. As this may take a long time to complete, it is critical to minimize the VM migration time so as to reduce the disruptions to the cloud services. In this article, a new scheduler, Raven, is proposed using an experiencedriven approach with deep reinforcement learning to schedule the virtual machine migration. A comparison of Raven with state-of-the-art algorithms shows that Raven can effectively shorten the time to complete the datacenter upgrade in different datacenter settings.

The eighth article entitled "Graph Neural Networks with Information Anchors for Node Representation Learning" presents a novel Graph Neural Network with Information Anchors for node embedding, called A-GNN model. In A-GNN, it incorporates the global structure information into the node vector, and fully utilizes the node features suitable for various network prediction tasks such as link prediction and node classification. The authors showed that A-GNN consistently outperforms existing baselines in link prediction and pairwise node classification tasks on five representative datasets.

The last article entitled "Jamming-Resilient Backup Nodes Selection for RPL-based Routing in Smart Grid AMI Networks" studies Advanced metering infrastructure (AMI), a core component of the smart grid. It conducts the analysis on the performance degradation problem of RPL protocol under the jamming attack. It proposes a backup node selection mechanism based on the standard RPL protocol. The proposed mechanism chooses a predefined number of backup nodes that maximize the probability of successful transmission. The evaluation results show that the proposed mechanism can greatly improve the performance of the standard RPL protocol under the jamming attack.

Acknowledgements The guest editors would like to thank the many people who made this Special Issue possible. We are especially grateful to all authors for their valuable contributions, and all the reviewers for their efforts in reviewing the manuscripts. We also thank the Edit-in-Chief, Prof. Imrich Chlamtac, for his supportive guidance during the entire process, as well as all the Editorial Staffs.

Publisher's note Springer Nature remains neutral with regard to jurisdictional claims in published maps and institutional affiliations.

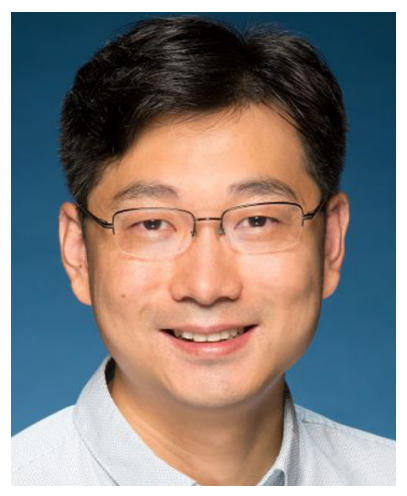

Xiaowen Chu received the B.E. degree in computer science from Tsinghua University, P.R. China, in 1999, and the Ph.D. degree in computer science from The Hong Kong University of Science and Technology in 2003. Currently, he is a full professor in the Department of Computer Science, Hong Kong Baptist University. His research interests include distributed and parallel computing and wireless networks. He served or is serving as an Associate Editor of IEEE Access and IEEE Internet of Things Journal, and Guest Editor of IEEE Transactions on Network Science and Engineering, IEEE Transactions on Industrial Informatics, Concurrency and Computation: Practice and Experience. He is a senior member of the IEEE.

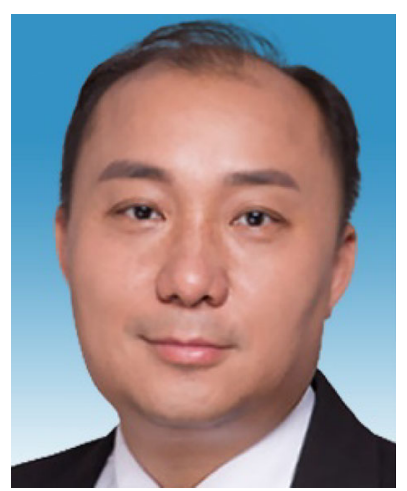

Hongbo Jiang is now a full Professor in the College of Computer Science and Electronic Engineering, Hunan University. He ever was a Professor at Huazhong University of Science and Technology. He received his $\mathrm{Ph} . \mathrm{D}$. from Case Western Reserve University in 2008. His research concerns computer networking, especially algorithms and protocols for wireless and mobile networks. He is serving as the Editor for IEEE/ACM Transactions on Networking, the Associate Editor for IEEE Transactions on Mobile Computing, and the Associate Editor for IEEE Internet of Things Journal. He is an elected Fellow of IET, Fellow of BCS, Senior Member of IEEE, Senior Member of ACM. 


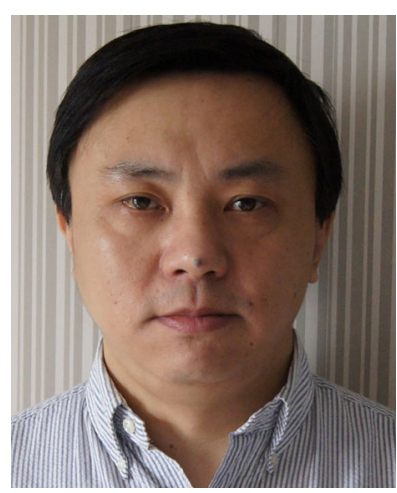

Bo $\mathbf{L i}$ is a Chair Professor in the Department of Computer Science and Engineering, Hong Kong University of Science and Technology. He held a Cheung Kong Visiting Chair Professor in Shanghai Jiao Tong University between 2010 and 2016, and was the Chief Technical Advisor for $\mathrm{Ch}$ i n a $\mathrm{C}$ a h e Corp . (NASDAQ:CCIH), a leading $\mathrm{CDN}$ provider. He was an adjunct researcher at the Microsoft Research Asia (MSRA) (19992006) and at the Microsoft Advanced Technology Center (2007-2008). He made pioneering contributions in multimedia communications and the Internet video broadcast, in particular the Coolstreaming system, which was credited as first largescale Peer-to-Peer live video streaming system in the world. It attracted significant attention from both industry and academia with over USD $25 \mathrm{M}$ VC fund, and received the Test-of-Time Best Paper Award from IEEE INFOCOM (2015). He has been an editor or a guest editor for over a two dozen of IEEE and ACM journals and magazines. He was the CoTPC Chair for IEEE INFOCOM 2004. He is a Fellow of IEEE. He received his $\mathrm{PhD}$ in the Electrical and Computer Engineering from University of Massachusetts at Amherst, and his B. Eng. (summa cum laude) in the Computer Science from Tsinghua University, Beijing, China.

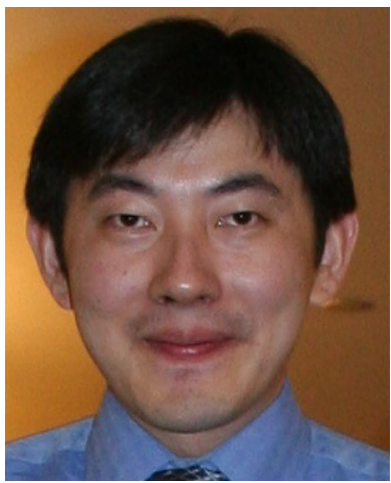

Dan Wang received the B.Sc. degree from Peking University, China, M.Sc. degree from Case Western Reserve University, USA, and Ph.D. degree from Simon Fraser University, all in computer science. Currently, he is an associate professor in the Department of Computing, The Hong Kong Polytechnic University. His research interests include computer networking and recently in smart energy systems. He served as TPC co-chairs of ACM e-Energy 2020 and

IEEE/ACM IWQoS 2020. He serves as an Associate Editor in IEEE Transactions on Mobile Computing. He is a senior member of the IEEE.

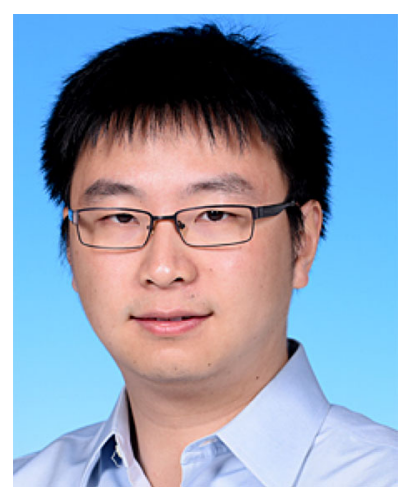

Wei Wang received the B.Eng. (Hons.) and M.Eng. degrees from Shanghai Jiao Tong University, and the Ph.D. degree from the University of Toronto in 2015, all in the Department of Electrical and Computer Engineering. Currently, he is an Assistant Professor in the Department of Computer Science and Engineering at the Hong Kong University of Science and Technology (HKUST). He is also affiliated with HKUST Big Data Institute. His research interests cover the broad area of distributed systems, with special emphasis on big data and machine learning systems, cloud computing, and computer networks. Dr. Wang served on the Program Committee of various prestigious international conferences and was recognized as the Distinguished TPC Member of IEEE INFOCOM 2018-20. He is a member of IEEE and ACM. 